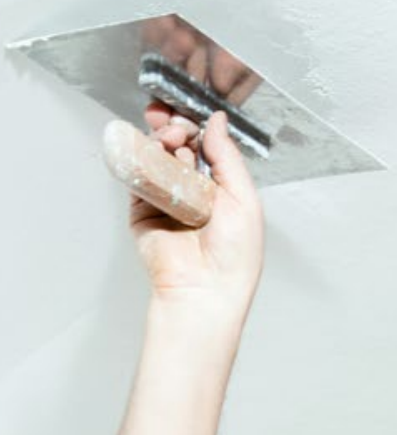

Welche Materialien und Techniken?

\title{
Befestigung von Glaskeramiken auf Molaren
}

\begin{abstract}
Zementierte Glaskeramiken sind beliebter denn je, insbesondere nachdem während der vergangenen Jahre neue Materialien, Klebesysteme und neue Zemente entwickelt worden sind. Auch für die Versorgung der Molaren wurden neue keramische Materialien entwickelt.
\end{abstract}

Dabei handelt es sich um Oxidkeramiken oder Glaskeramiken. Die Glaskeramiken sind besonders interessant, da sie Silikat enthalten und ihre Mikrostruktur eine adhäsive Befestigung zu Schmelz und Dentin erlauben. Daher können Glaskeramiken starken Kräften, wie sie im Molarenbereich auftreten, widerstehen, ohne dass der Zement nachgibt. Das gilt ebenso für nichtretinierende Kronenpräparationen. Seitdem auch die Oberfläche von Feldspatkeramiken modifiziert werden können, sind Materialien höchster Widerstandskraft und Ästhetik erhältlich. Mit Hilfe eines modifizierten Brennalgorithmus kann auch die Materialfestigkeit dieser Keramiken gesteigert werden.

Zur adäquaten Befestigung der Restauration auf dem Zahnstumpf sind üblicherweise eine Modifikation der Keramikoberfläche sowie auch des Schmelzes oder des Dentins notwendig. Von den Befestigungszementen werden ein guter Abschluss am Kronenrand und die Bewahrung der originären Farbe gefordert. Die ersten Zemente waren Glasionomerzemente oder basierten auf Zinkphosphatzusammensetzungen. Mit der Einführung von Polymeren (Resinen) als Befestigungsmaterialien wurden die Eigenschaften des Auswaschens über die Zeit, der Löslichkeit und der Adhäsion verbessert.

\section{Verschluss der Dentinkanälchen mit adhäsivem Resin} Vor der Abformung des präparierten Zahnstumpfes wird zudem ein Verschluss der Dentinkanälchen mit einem adhäsiven Resin empfohlen, um später eine stärkere Adhäsion der Krone, einen geringeren Spalt und verminderte Dentinsensibilität zu erreichen. Verschiedene Autoren haben dieses „immediate den- tin sealing" empfohlen (zum Beispiel Pashley). Die Verwendung von Polymer-Resinen ist allerdings aufwändiger, da eine Trockenhaltung des Zahnes erforderlich ist.

Im Ergebnis einer Metaanalyse von Breemer et al. (2015) scheinen adhäsive Resin-basierte Produkte mit einer Ätz- und Spültechnik in drei Schritten den höchsten Scherkräften widerstehen zu können und sind damit den selbstadhäsiven und selbstätzenden Systemen überlegen. Was die Befestigung neuer Keramiken wie der Lithiumdisilikatkeramik angeht, liegen aktuell nur wenige Studien vor. Bislang wurde bezüglich der Befestigungsmodalität dieser Materialien kein Material oder Befestigungsprozess präferiert.

Allein die sogenannte „Immediate-dentin-sealing“-Technik scheint die Zugfestigkeit bei allen Keramikmaterialien zu steigern. Allerdings basieren diese Erkenntnisse auf In-vitro-Experimenten und sind klinisch noch nicht verifiziert worden.

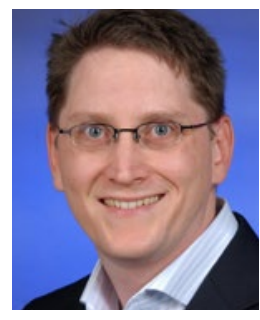

Prof. Dr. Dr. Felix P. Koch

Autor des Wissenschaftlichen Infodienstes, Lehrbeauftragter der Uniklinik Frankfurt, Praxisklinik für Mund-, Kiefer- und plastische Gesichtschirurgie in Wiesbaden. Mail:wid@fvdz.de 